\title{
Polarized Single Photon Emission for Quantum Cryptography Based on Colloidal Nanocrystals
}

\author{
Ferruccio Pisanello ${ }^{1,2}$, Luigi Martiradonna ${ }^{1}$, Piernicola Spinicelli ${ }^{2}$, Angela Fiore ${ }^{2}$, Jean-Pierre Hermier ${ }^{3}$, \\ Liberato Manna ${ }^{1}$, Roberto Cingolani ${ }^{1}$, Elisabeth Giacobino ${ }^{2}$, Alberto Bramati ${ }^{2}$ and Massimo De Vittorio ${ }^{1}$ \\ ${ }^{I}$ National Nanotechnology Laboratory, CNR/INFM, Scuola superiore ISUFI, Università del Salento \\ 16 Via Arnesano, Lecce 73100, Italy \\ ${ }^{2}$ Laboratoire Kastler Brossel, CNRS UMR8552, Universitè Pierre et Marie Curie, Ecole Normale Supérieure \\ 4 place Jussieu, 75252 Paris Cedex 05, France \\ ${ }^{3}$ Groupe d'etude de la Matière Condensée, CNRS UMR8635, Universitè de Versailles \\ Saint-Quentin-en-Yvelines, 45 avenue de Etas-Unis, 78035 Versailles Cedex, France
}

\begin{abstract}
In this paper, the evidence of a polarized and room temperature single photon emission from wet-chemically synthesized colloidal dot-in-a-rod is reported. The time and polarization resolved measurements clearly indicate a high degree of linear polarization and a lifetime of $\sim 11 \mathrm{~ns}$. We report also about a viable strategy to develop single photon sources with polarization control for quantum cryptography.
\end{abstract}

Keywords: single photon sources, colloidal nanocrystals, quantum cryptography.

\section{INTRODUCTION}

Quantum networking and quantum cryptography are recently subject of intense research activity. Quantum information technology (qIT) algorithms are usually based on antibunched or entangled photon fluxes and on the fine control of their quantum states and polarization properties. For instance, in BB84 and B92 algorithms [1,2] for secure exchange of a private cryptography key, sender and receiver exchange keys by means of a flux of single photon having a controlled polarization. Several sources for qIT were developed in past years by using fluorescence from a single atom in a cavity, defects in diamonds and artificial atoms. A promising alternative relies on single photon emission from a single quantum dot (QD). Nowadays, two different technologies are in competition for developing high efficiency QD-based single photon sources (SPSs): Stranski-Krastanov epitaxial QDs and wet-chemically synthesized colloidal nanocrystals (NCs).

By virtue of the possibility to simply achieve electrical injection, epitaxial QDs are actually the most investigated nanostructures for qIT applications. The concept of QDs was initially proposed by Arakawa et al. in 1982 [3] and, in the last years, several studies were carried out in order to use these QDs as source of single or entangled photons. SPS behaviour at a relatively high temperature (T 200 K) was presented in 2002 [4] for a single epitaxial QD and in 2008 for core/shell epitaxial structures (T 220 K) in which a single QD was embedded in an epitaxial quantum wire [5]. However, room temperature single photon emission in epitaxially grown QD has not been demonstrated, so far.

On the other hand, by virtue of the higher distances between the allowed energy states in both conduction and valence bands, colloidal core/shell NCs show an high emission efficiency and single photon behaviour also at room temperature[6]. Due to their versatile and low cost synthesis procedures, high quantum yield and broad tunable emission range [7,8], colloidal NCs are considered the most promising alternative to epitaxial QDs for qIT applications. The first evidences of non classical light emitted from colloidal NCs were reported in the last few years $[6,9,10]$.

So far, NCs applications have been limited by some drawbacks, such as blinking, spectral diffusion, long luminescence lifetime and unpolarized and uncollimated emission [12,13]. Recently, it has been demonstrated that the shape and size of the NCs can be tailored to overcome these limitations: almost not blinking QDs capped by several shell layers were proposed in ref. [14], while polarized emission was detected by elongated structures such as core nanorods [15] and core/shell nanorods [16]. Moreover, by coupling the nanocrystals with a confined mode in cavities structures an increased spontaneous emission rate was shown by virtue of the Purcell effect [17, 18]. Among the engineered nanoclusters proposed so far, dot-in-rod (DRs) nanocrystals obtained by growing a CdS rod-like shell onto a spherical CdSe QD [19,20] are now catching the scientific community interest. Thorough studies about their photophysical properties are revealing peculiar characteristics such as strongly localized wavefunctions for both electrons and holes in the CdSe core [21,22], high photoluminescence efficiency and giant extinction coefficients in the UV range [20], strong dipole moment which allows to control the DR alignment on flat surfaces by applying a localized electric field [19]. Moreover, polarized emission has been detected from aligned ensembles of DR [19]. These peculiarities let us envision the application of these emitters in polarization controlled, low cost and highly efficient room temperature single photon sources, although the non-classical behaviour of single DRs has not been completely investigated yet. In this sense, the spectroscopic analysis performed in this work is meant to collect further knowledge on these nano-objects, by analysing the antibunching behaviour and the polarization properties of a single CdSe/CdS DR emission. 

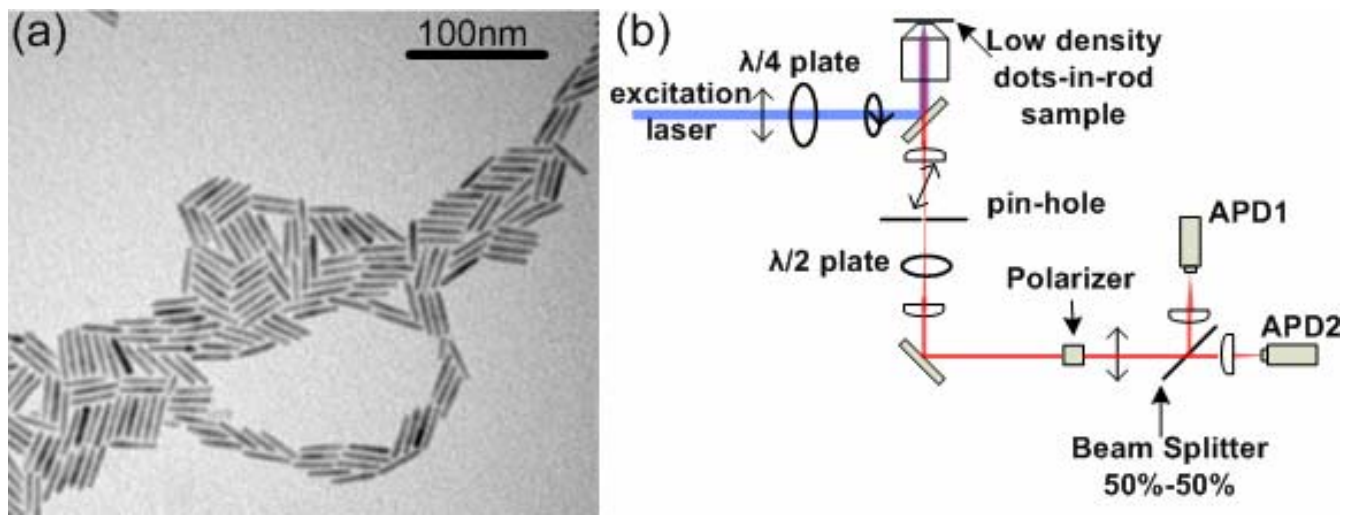

Figure 1. (a)Transmission electron microscope image of a typical output of the seeded growth synthesis. (b) Experimental setup used to carry out spatially polarized and time resolved measurements.

\section{METHODS}

\subsection{Sample preparation}

The $\mathrm{CdSe} / \mathrm{CdS}$ core/shell dot in rod nanocrystals have been synthesized with a seeded growth approach [19], which yields nanorods with narrow distributions of both lengths and diameters. The synthesis is based on the coinjection of preformed spherical CdSe nanocrystal seeds and chemical precursors into a hot mixture of surfactants suited for the anisotropic growth of CdS nanocrystals. A typical output of the synthesis process is reported in Fig. 1a. A mono-molar solution (in toluene) was drop-casted on a microscope glass coverslip for single nanocrystal spectroscopy.

\subsection{Optical characterization}

The spatial, polarization and time resolved analyses were performed by using the confocal microscopy configuration reported in Fig. 1b. The DRs were excited by means of a circularly polarized laser with $\sim 100 \mathrm{ps}$ pulses, focused on to a single DR by an high numerical aperture (N.A. 0.7) objective. In order to scan the sample surface and to identify isolated single NCs, the objective was fixed on a piezoelectric motor. Fluorescence from single emitters was collected by using the same objective and spatially filtered by a size-tunable pin-hole. The light from the pin-hole was directed to a polarization resolved section, based on an half wavelength plate and on a linear polarizer, which allowed us to analyze the properties of the emitted photons for several polarization angles $(\theta)$. The output of the polarizer was sent to an high sensitivity Hanbury-Brown and Twiss setup, based on two avalanche photodiodes (indicated with APD1 and APD2 in Fig. 1b). The signal detected by the photodiodes was analyzed by using a time resolved data acquisition card (TimeHarp200), based on two acquisition functions. The start/stop mode measures the distance between two received photons with a time resolution of $\sim 1.1 \mathrm{ns,}$ building the coincidence histogram in a $4.7 \mu$ s window, which, being proportional to the autocorrelation function $g^{(2)}(\tau)$, was used to verify the single photon behaviour. The laser triggered mode was instead used to record both the individual photon events, with their absolute arrivals times, and the delays from the laser pulses, and it was employed in order to measure the decay curve with a time resolution of $\sim 150 \mathrm{ps}$.

\section{RESULTS AND DISCUSSIONS}

As reported in ref. [19], the maximum photoluminescence intensity is detected by collecting the light polarized along the rod axis, i.e. for $\theta=0^{\circ}$. The emission for $\theta=0^{\circ}$ is characterized by two intensity levels, hereafter referred to as ON state, when the DR is emitting light, and OFF state, in which the collected signal is comparable to the noise level, because of the NC blinking. The statistical average values of the ON state, for several polarization angles, are reported in Fig. 2a. The emission intensity follows a $\cos ^{2}(\theta)$ behaviour, which clearly indicates the polarized nature of the light emitted from a single dot in rod. The calculated degree of linear polarization $\left(I_{\|}-I_{\perp}\right) /\left(I_{\|}+I_{\perp}\right)$ corresponds to $75 \%$.

In order to proof the single photon source behaviour of single colloidal DRs, the coincidence histogram was measured by using the start/stop mode for several isolated DR, and a typical result is shown in Fig. $2 \mathrm{~b}$. The small number of coincidence events around zero delay time indicates that the probability to detect two photons at the same time is extremely low, confirming the SPS behaviour of the DRs. On the other hand, the sharp periodic peaks are the evidence that the detected photons are usually spaced by a multiple of the period of the laser pulses. 
The electron-hole pair lifetime was measured by using the histogram of the photon delays from the laser pulses. By using the laser triggered mode we obtained the logarithmic decay curve reported in Fig. 2c, which was fit by a mono-exponential function $A_{r} e^{-\left(t-t_{0}\right) / \tau_{r}}$, obtaining a $\tau_{r} \sim 11.1 \mathrm{~ns}$.
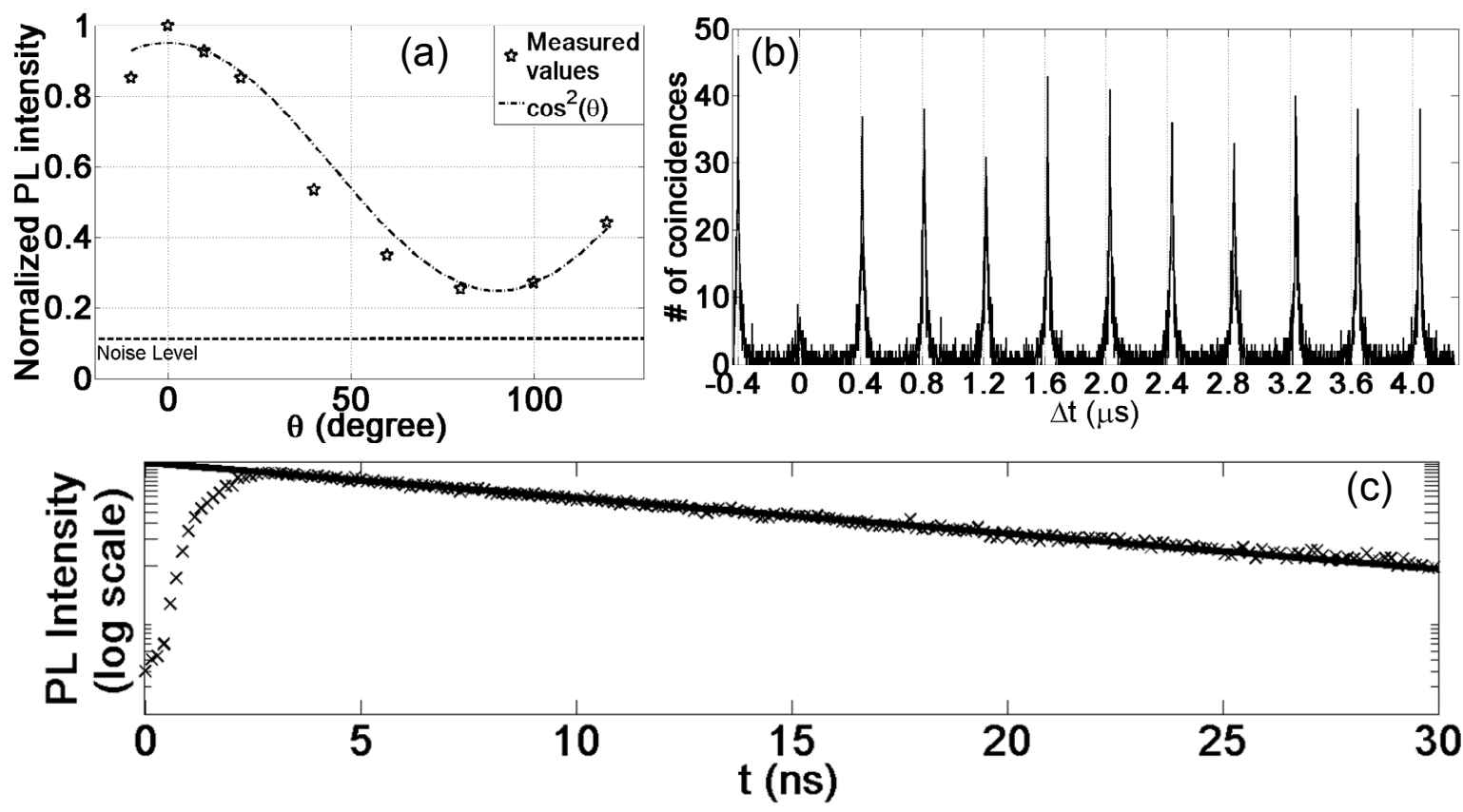

Figure 2. (a) Normalized photoluminescence for several polarization detection angles. (b) Coincidence histogram collected from a single DR. (c) Decay curve obtained by collecting the signal in laser triggered mode.

The control of the polarization of a single photon flux is a key issue in the optimization and implementation of quantum cryptography algorithms. The table reported in Fig. 3 shows a typical example of the implementation of the BB84 algorithm. Alice sends to Bob a flux of bits by randomly choosing (for each bit) between two different polarization bases and Bob receives and extracts the bits by independently choosing his bases. After the public declaration of their basis, but NOT of the sent and received bits, the cryptography key is derived by extracting the bits sent and received with the same bases.

The use of dot-in-rod single nanocrystals would make possible to emit single photons with the desired polarization basis by orienting the nanocrystals at $0^{\circ}, 90^{\circ},-45^{\circ}, 45^{\circ}$ with respect to a specific axis. A defined orientation of the DRs can be achieved by different methods, such as by using a strong electric field [19], by mechanically moving the rods through MEMS nanoactuators or by localizing and orienting single nanocrystals on prepatterned substrates [17]. The use of specifically oriented polarized emitters allow us to avoid the recourse to optical components, such as linear polarizers, which would affect the source rate.

\begin{tabular}{|c|c|c|c|c|c|c|c|c|c|c|c|c|}
\hline Sample \# & 1 & 2 & 3 & 4 & 5 & 6 & 7 & 8 & 9 & 10 & 11 & 12 \\
\hline Random bit & 1 & 0 & 0 & 0 & 1 & 0 & 1 & 1 & 0 & 0 & 1 & 0 \\
\hline Random sending base & $x$ & + & + & $x$ & $x$ & $x$ & + & $x$ & $x$ & + & $x$ & $x$ \\
\hline Sent photon polarization & $\swarrow$ & $\leftrightarrow$ & $\leftrightarrow$ & $\nwarrow$ & $\nearrow$ & $\nwarrow$ & $\uparrow$ & $\swarrow$ & ฐ & $\longleftrightarrow$ & $\nearrow$ & ฐ \\
\hline Random receiving base & + & + & $x$ & $x$ & $x$ & + & + & + & + & + & $x$ & + \\
\hline Received photon polarization & & $\leftrightarrow$ & $\nearrow$ & $\nwarrow$ & $\nearrow$ & $\leftrightarrow$ & $\uparrow$ & & $\leftarrow$ & $\leftrightarrow$ & $\nearrow$ & $\leftrightarrow$ \\
\hline Received bit & 0 & 0 & 1 & 0 & 1 & 0 & 1 & 0 & 0 & 0 & 1 & 0 \\
\hline Bases AND & & $\checkmark$ & & $\checkmark$ & $\checkmark$ & & $\checkmark$ & & & $\checkmark$ & $\checkmark$ & \\
\hline Shared key & & 0 & & 0 & 1 & & 1 & & & 0 & 1 & \\
\hline
\end{tabular}

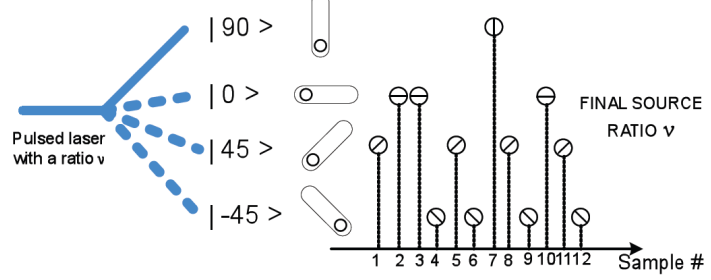

Figure 3. Implementation of the BB84 algorithm by using DRs.

\section{CONCLUSIONS}

In summary we reported the evidence of a polarized single photon flux from a colloidal dot-in-rod by using a time and polarization resolved spectroscopy. Exhibiting a degree of linear polarization of $\sim 75 \%$, our polarized nanoparticles could be exploited for room-temperature quantum cryptography in communication systems. 


\section{ACKNOWLEDGEMENTS}

We thank Rosanna Mastria and Benedetta Antonazzo for their support in the synthesis of colloidal nanocrystals, Gianmichele Epifani and Gianvito De Iaco for their expert technical help, Riccardo Messina, Antonio Qualtieri, Dr. Giovanni Morello, Dr. Alberto Amo and Dr. Giuseppe Patera for useful discussions and Trong Chang Nguyen and Godefroy Lemenager for the help in the measurements.

\section{REFERENCES}

[1] C. H. Bennet and G. Brassard: Quantum Cryptography: Public key distribution and coin tossing, in Proc. of The IEEE International Conference on Computers, Systems, and Signal Processing, Bangalore, India, p. 175-179 (1984).

[2] C. H. Bennet: Quantum cryptography using any two nonorthogonal states, Phys. Rev. Lett, vol. 68, pp. 3121-3124, 1992.

[3] Y. Arakawa and H. Sakaki: Multidimensional quantum well laser and temperature dependence of its threshold current, Appl. Phys. Lett., vol. 40, pp. 939-941, Jun. 1982.

[4] C. Santori, K. Hoshino, S. GÖtzinger, Y. Yamamoto, S. Kako, Y. Arakawa: A gallium nitride singlephoton source operating at $200 \mathrm{~K}$, Nat. Mat., vol. 5 pp. 887-893, Oct. 2006.

[5] A. Tribu et al:: A High-Temperature Single-Photon Source from Nanowire Quantum Dots, Nano Lett., vol. 8, pp. 4326-4329, Nov. 2008.

[6] P. Michler, A. Imamoglu, M. D. Mason, P. J. Carson, G. F. Strouse and S.K. Buratto: Quantum correlation among photons from a single quantum dot at room temperature, Nature, vol. 406 pp. 968-970, Aug. 2000.

[7] C. B. Murray, D. J. Norris and M. G. Bawendi: Synthesis and characterization of nearly monodisperse CdE $(\mathrm{E}=$ sulfur, selenium, tellurium) semiconductor nanocrystallites, J. Am. Chem. Soc., vol. 115 pp. 87068715, Sept.1993.

[8] X. Brokmann, L. Coolen, M. Dahan and J. P. Hermier: Measurement of the radiative and nonradiative decay rates of single CdSe nanocrystals through a controlled modification of their spontaneous emission, Phys. Rev. Lett., vol. 93, pp. 1074031-1074034, 2004.

[9] B. Lounis, H. A. Bechtel, D. Gerion, P. Alivisatos and W. E. Moerner: Photon antibunching in single CdSe/ZnS quantum dot fluorescence, Chem. Phys. Lett., vol. 329 pp. 399-404, Dec. 2000.

[10] G. Messin, J. P. Hermier, E. Giacobino, P. Desbiolles, and M. Dahan: Bunching and antibunching in the fluorescence of semiconductor nanocrystals, Opt. Lett., vol. 26, pp. 1891-1893, Dec. 2001.

[11] K. L. Knappenberger Jr. et al.: Excitation-wavelength dependence of fluorescence intermittency in CdSe nanorods, ACS Nano, vol. 2, pp. 2143-2153, Sept. 2008.

[12] R. G. Neuhauser, K. T. Shimizu, W. K. Woo, S. A. Empedocles and M. G. Bawendi: Correlation between fluorescence intermittency and spectral diffusion in single semiconductor quantum dots, Phys. Rev. Lett., vol. 85, pp. 3301-3304, Apr. 2000

[13] G. Schlegel, J. Bohnenberger, I. Potapova and A. Mews: Fluorescence decay time of single semiconductor nanocrystals, Phys. Rev. Lett., vol. 88, pp. 1374011-1374014, Mar. 2002.

[14] B. Mahler et al.: Towards non-blinking colloidal quantum dots, Nature Mat., vol. 7 pp. 659-664, Jun. 2008.

[15] J. Hu, L. S. Li, W. Yang, L. Manna, L. W. Wang, A. P. Alivisatos: Linearly polarized emission from colloidal semiconductor quantum rods, Science, vol. 292, pp. 260-263, Apr. 2001.

[16] N. Le Thomas, E. Herz, O. Schops., U.Woggon and M.V. Artemyev: Exciton fine structure in single CdSe nanorods, Phys. Rev. Lett., vol. 94, pp. 0168031-0168034, Jan. 2005.

[17] A. Qualtieri et al.: Nonclassical emission from single colloidal nanocrystals in a microcavity: a route towards room temperature single photon sources, New J. Phys., vol. 11, pp. 033052-033063, Mar. 2009.

[18] N. Le Thomas et al.: Cavity QED with Semiconductor nanocrystals, Nano Lett., vol. 6 pp. 557-561, Feb. 2006.

[19] L. Carbone et al.: Synthesis and micrometer-scale assembly of colloidal CdSe/CdS nanorods prepared by a seeded growth approach, Nano Letters, vol. 7, pp. 2942-2950, Jun. 2007.

[20] D. V. Talapin et al: Seeded growth of highly luminescent CdSe/CdS nanoheterostructures with rod and tetrapod morphologies, Nano Lett., vol. 7, pp. 2951-2959, Sept. 2007.

[21] D. Steiner et al:: Determination of Band Offsets in Heterostructured colloidal nanorods using scanning tunneling spectroscopy, Nano Lett., vol. 8, pp. 2954-2958, Aug. 2008.

[22] G. Morello et al.: Intrinsic optical nonlinearity in colloidal seeded grown CdSe/CdS nanostructures: Photoinduced screening of the internal electric field, Rev. B, vol. 78 195313-195313, Nov. 2008. 